\title{
Synchrotron diffraction study of the cementite phase in cold drawn pearlitic steel wires \\ Jeroen Tacq ${ }^{1, a}$, Martin Kriška ${ }^{1, b}$ and Marc Seefeldt ${ }^{1, c}$
}

${ }^{1}$ Catholic University of Leuven, Department of Metallurgy and Materials Engineering,

B-3001 Heverlee (Leuven), Belgium

ajeroen.tacq@mtm.kuleuven.be, ${ }^{b}$ martin.kriska@mtm.kuleuven.be,

cmarc.seefeldt@mtm.kuleuven.be

Published by Trans Tech Publications (http://www.ttp.net)

Materials Science Forum, International Conference on Residual Stresses 9 (ICRS 9), pp. 380 (2014)

This is the authors personal copy.

The research was funded by Jeroen Tacq 's fellowship of the Fund for Scientific Research-Flanders (FWO-V) and supported by the Belgian Science Policy BELSPO through the IAP project 7/21. 


\title{
Synchrotron diffraction study of the cementite phase in cold drawn pearlitic steel wires
}

\author{
Jeroen Tacq ${ }^{1, a}$, Martin Kriška ${ }^{1, b}$ and Marc Seefeldt ${ }^{1, c}$ \\ ${ }^{1}$ Catholic University of Leuven, Department of Metallurgy and Materials Engineering, \\ B-3001 Heverlee (Leuven), Belgium \\ ajeroen.tacq@mtm.kuleuven.be, ${ }^{b}$ martin.kriska@mtm.kuleuven.be, \\ cmarc.seefeldt@mtm.kuleuven.be
}

\section{Keywords: Pearlite, Cementite, Texture, Residual stress, Synchrotron Diffraction}

\begin{abstract}
Energy dispersive synchrotron diffraction has been carried out on cold drawn pearlitic steel wires. In this paper the observed cementite peaks are analysed. For a broad range of true drawing strains $\sin ^{2}(\psi)$ curves have been measured. The residual stress in the cementite is found to saturate after reaching a maximum at a strain of about 1.6. No indication of significant texture development in the cementite could be observed. An explanation is given in terms of possible physical mechanisms. Peak broadening was observed at the early stages of deformation.
\end{abstract}

\section{Introduction}

Pearlitic steel wire is a two phase material having a lamellar microstructure, produced by wire drawing. The wire drawing process results in an alignment of the lamellae with the drawing axis and consequent thinning and curling of the lamellae [1,2]. This behaviour results in an exponential strengthening of the wire with increasing drawing strain, giving rise to very high tensile strengths $[3,4]$. At high strain the cementite phase is known to decompose [5-7], leading to an increase of the carbon content in the ferrite phase. The carbon is mainly clustered around lattice defects $[8,9]$.

The excellent properties of pearlitic steel wires make them perfect for many industrial applications such as cables for bridges and sawing wires for wafers. A lot of research has already been expended on the study of the influence of residual stress, texture and the exact microstructural features of the pearlite on the mechanical properties of the wire. To this day, a full understanding of the behaviour of the pearlitic steel has however still not been achieved.

The study of pearlite has mainly been focussed on the ferrite phase for a long time. The cementite was considered to be elastically isotropic. However, it has now been shown that the cementite phase exhibits (strong) elastic anisotropy [10,11]. It is also widely accepted that the cementite deforms plastically during wire drawing [2,12]. Consequently, plastic anisotropy of cementite might lead to a texture development.

Ferrite and cementite crystallographic orientation, together with the morphological orientation (i.e. alignment of the lamellae with respect to an external reference frame) determine the phase boundary structure. The structure of the phase boundary determines its efficiency as a dislocation source or sink and has an influence on the stability of the cementite phase [13].

This study aims at contributing to the understanding of the pearlite behaviour by a detailed analysis of the cementite peaks recorded during residual stress measurements. Next to the measured residual stress, special attention will be paid to the possible existence of a cementite texture and cementite peak broadening. The main concern of the experiment were the residual stresses in the ferrite phase [14]. The quality of the data obtained on the cementite phase is therefore not entirely satisfactory. However, an effort is made to learn as much as possible from the available data. 


\section{Material and experimental methods}

The material used in the current investigation is a hypereutectoid steel wire having a microstructure consisting of ferrite and cementite lamellae. The wire contains about $0.92 \mathrm{wt} \% \mathrm{C}$, $0.36 \mathrm{wt} \% \mathrm{Mn}$ and $0.21 \mathrm{wt} \% \mathrm{Si}$. The cementite phase has an orthorhombic crystal structure $(\mathrm{a}=$ $5.084, \mathrm{~b}=6.742, \mathrm{c}=4.514$ ) and takes up about $16 \mathrm{vol} \%$ of the material. The steel wire has been cold drawn to various strains, up to a true drawing strain of 3.7. The as-patented wire has a thickness of $890 \mu \mathrm{m}$ and a thin brass coating of about $1.1 \mu \mathrm{m}$ thickness.

Diffraction patterns were collected from wires drawn to different strains using energy dispersive synchrotron radiation. The measurements have been carried out at the Helmholtz Zentrum Berlin (HZB) using the EDDI beam line [15]. They were performed using a diffraction angle $2 \theta$ of $8^{\circ}$. For each strain, 42 patterns are recorded: two angles of $\varphi\left(0^{\circ}\right.$ and $\left.90^{\circ}\right)$ and 21 angles of $\psi$. The diffracting volume of one wire is very small. To remedy this, a number of wires is placed next to each other. This is illustrated in Fig. 1, together with the definition of the angles. The residual stress in both the axial and transversal direction can be determined from the slopes of $d-\sin ^{2} \psi$ curves.

The beam current and thus intensity of the synchrotron radiation varied with time. At regular intervals a bunch of electrons is injected into the beam causing the beam current to increase. After each injection the beam current drops exponentially until the next injection. Measured intensities thus have to be corrected for the beam current. On top of this correction it should be held in mind that small peaks will be more difficult to resolve at low beam current.

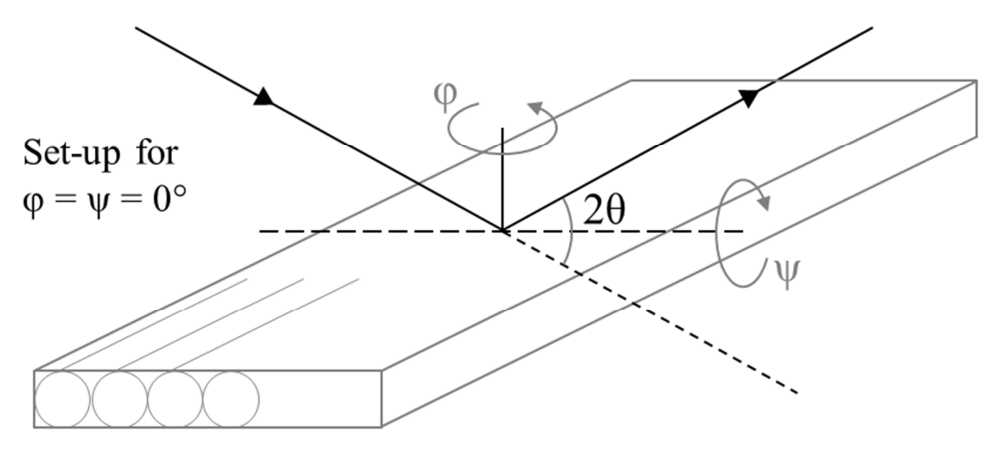

Fig. 1. Illustration of the experimental set-up.

The used set-up can also be used to get an indication of texture. Consider the horizontal axis of the pole figure to be parallel to the wire axis. The intensity along the horizontal axis of the pole figure can then be measured by varying $\psi$ from $0^{\circ}$ to $90^{\circ}$, for $\varphi$ equal to $0^{\circ}$. Varying $\psi$ for $\varphi$ equal to $90^{\circ}$ yields the intensity distribution along the vertical axis of the pole figure.

\section{Results}

The sharpest peaks in the measured diffraction patterns belong to the ferrite phase. From these peaks the ferrite residual stresses and peak broadening could be obtained. The results are discussed in two separate contributions to these proceedings by M. Kriška et al. [14] and M. Seefeldt et al. [16], respectively.

As-patented wire The entire diffraction pattern for the as-patented wire is shown in Fig. 2. The three highest peaks are, in order of increasing energy, the ferrite 110, 200 and 211 reflections. The counts have been cut off at 1000, the ferrite 110 peak has an intensity of around 5500 counts. The peaks at 87.55 and $97.86 \mathrm{keV}$ are the ferrite 220 and 310 reflection respectively. The larger peak at $34 \mathrm{keV}$ (90 counts) and smaller peak at $32.8 \mathrm{keV}$ (30 counts) are escape peaks related to the 110 ferrite peak. The small peaks at energies below $10 \mathrm{keV}$ are not caused by diffraction but by X-ray fluorescence and are as such of no interest to the current investigation.

Around the 110 ferrite peak some smaller peaks can clearly be observed. A detail of the pattern around the 110 ferrite peak is shown in Fig. 3. Weisser et al. [17] measured a much sharper 
diffraction pattern of cementite in bainite using synchrotron radiation. Some of the peak positions found by Weisser are indicated with lines in Fig. 3. Where peaks overlap too much, no separate lines have been drawn. A comparison of the profile with the indicated cementite peak positions shows that most of the peaks can be identified as cementite. However there is one peak with a dspacing of $2.133 \AA$ that could not be attributed to any of the allowed cementite reflections.

This extra peak can be attributed to the thin brass coating covering the wire. The observed dspacing corresponds to the 111 reflection of fcc $\alpha$-brass with lattice parameter $a=3.694 \AA$. If the stress in the coating is considered to be negligible for the as-patented wire, the measured lattice parameter corresponds to a zinc content of about $36 \mathrm{wt} \%$ [18]. This peak is discussed in some more detail in a further section of this paper.

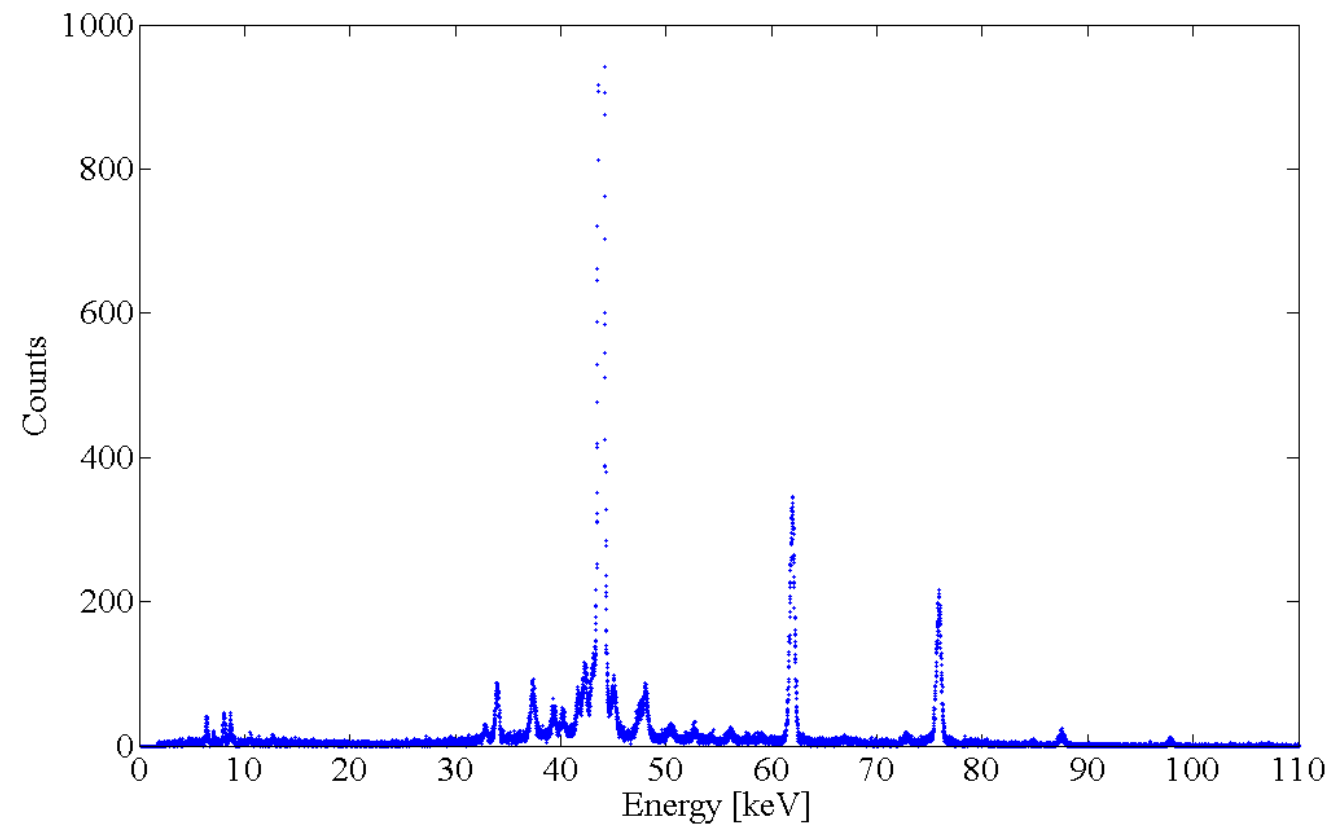

Fig. 2. Energy dispersive synchrotron diffraction spectrum of the as-patented wire $\left(\phi=\psi=0^{\circ}\right)$.

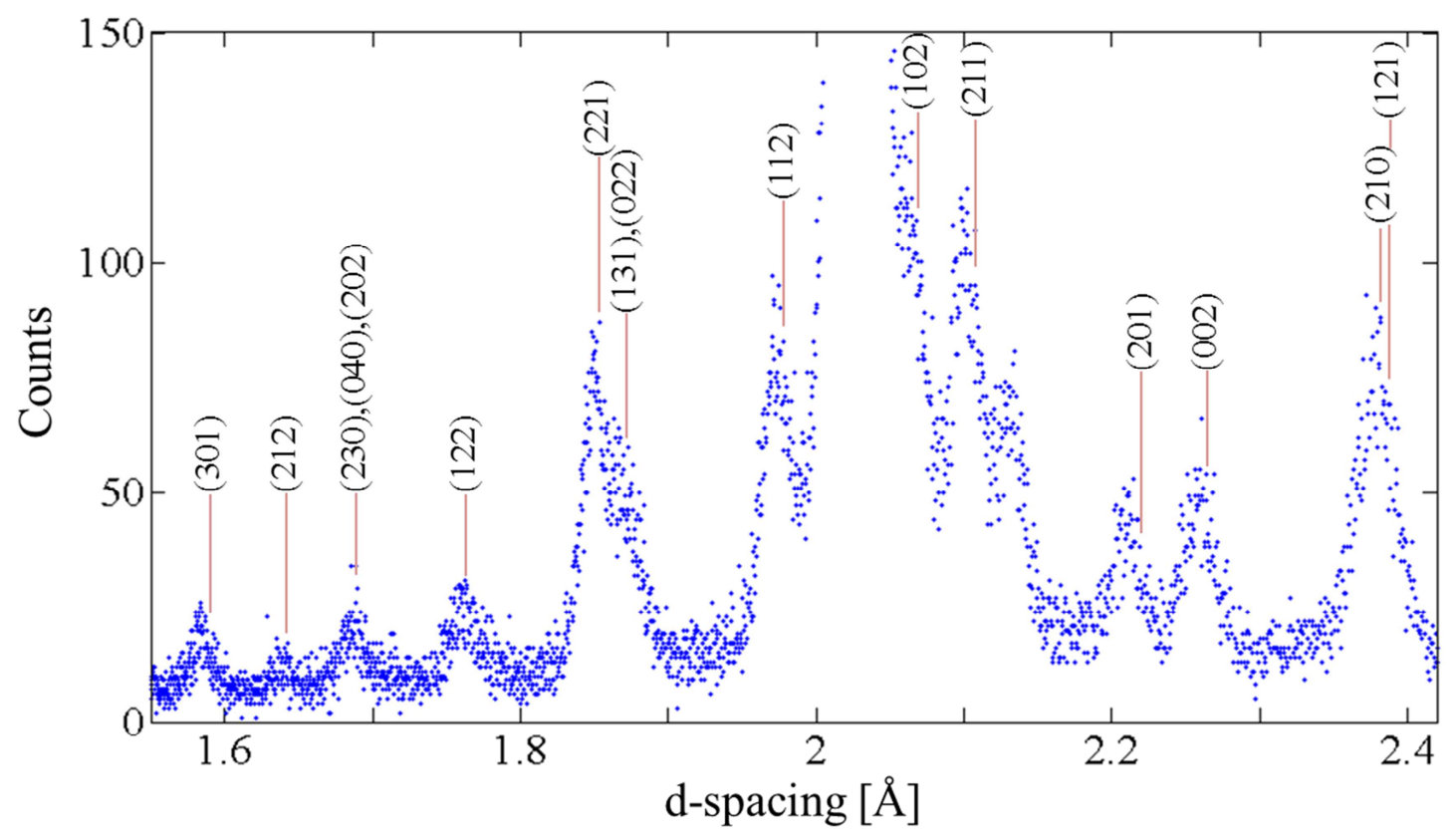

Fig. 3. Detail of the diffraction spectrum of the as-patented wire around the 110 ferrite peak. Energy values have been converted into d-spacings. 
True drawing strain 0.4-1.2 A close-up of the diffraction patterns $\left(\varphi, \psi=0^{\circ}\right)$ for wires cold drawn to true strains of 0 up to 1.2 is shown in Fig. 4. At a true strain of 0.4 the peaks on the high-d-side of the 110 ferrite peak become much broader and begin to overlap. The patterns for a true strain of 0 and 0.4 were measured at comparable beam currents. The beam currents during measurement of the patterns for wires with true strains of 0.6 and 1.2 were only half as high. The peaks on the high $\mathrm{d}$ side now almost seem to disappear completely. The patterns at a higher $\psi$ angle (for $\varepsilon=0.6$ and 1.2) were again measured at a higher beam current. Even then the peaks could still not be discerned unambiguously. Only the brass peak remains visible. It strongly overlaps with the 110 ferrite peak so that it manifests itself as a strong asymmetry of the ferrite peak towards the high-d-side.

On the low-d-side the maximum intensity of the peak around $1.85 \AA$ also strongly decreases. This peak seems to stabilise at a true strain of 0.6 and remains more or less visible, depending on the beam current. The software provided at HZB was able to fit this peak and determine the peak position. It should be held in mind that this peak is a combination of three cementite reflections (221, 131 and 022).

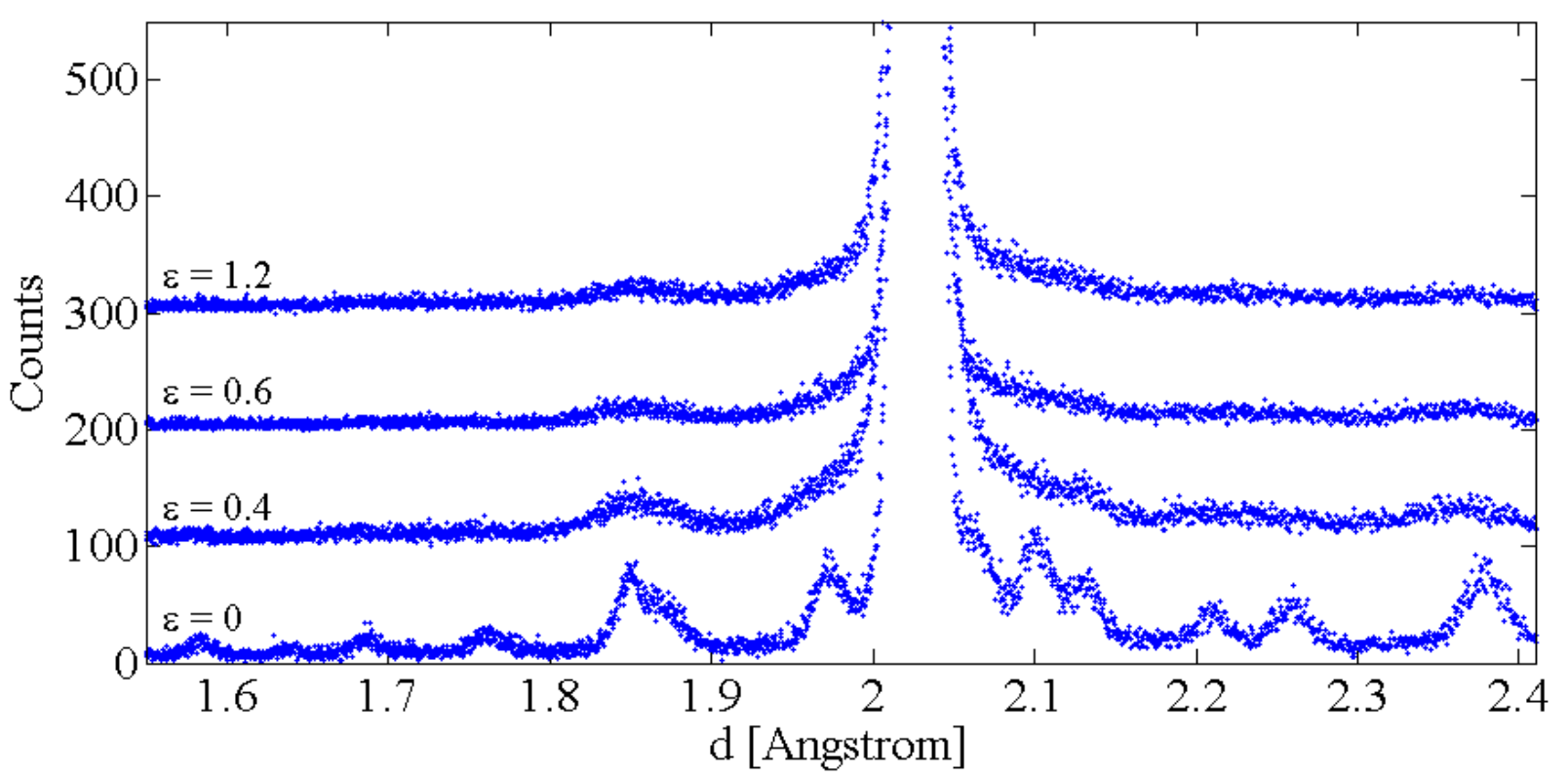

Fig. 4. Diffraction spectra $\left(\phi=\psi=0^{\circ}\right)$ measured on wires drawn to different strains $(0,0.4,0.6$ and 1.2).

Residual stress At still higher strains, accurately determining the peak position of the peak around $1.85 \AA$ becomes more difficult. Patterns were collected for many $\psi$ angles and the peak positions determined. After a selection of the reliable peak positions, which coincides with high beam currents, enough points were left in the $\mathrm{d}-\sin ^{2} \psi$ curves to determine the residual stresses in the axial direction up to a true train of 2.8. The cementite axial stresses are shown in Fig. 5. The stress in the transverse direction is always a mixture of hoop and radial stresses and was zero within the accuracy of the current experiment. The transverse stresses will therefore not be discussed further. The discussion further in this paper shows that the stress calculation is reliable, even though the used peak consists of three overlapping reflections.

At higher strains the 110 ferrite peak exhibits a strong asymmetry towards the high-d-side for patterns collected where $\varphi$ is $0^{\circ}$. For $\varphi$ equal to $90^{\circ}$ this asymmetry is not present, however the peak around $2.13 \AA$ is again visible. The patterns for a true drawing strain of 2.1 and $\psi=30^{\circ}$ are shown in Fig. 6. The difference between the two patterns can be explained as the brass peak having almost no stress in the transverse direction while being under compression in the axial direction, shifting the peak to a smaller d-spacing and causing strong overlap with the 110 ferrite peak. 


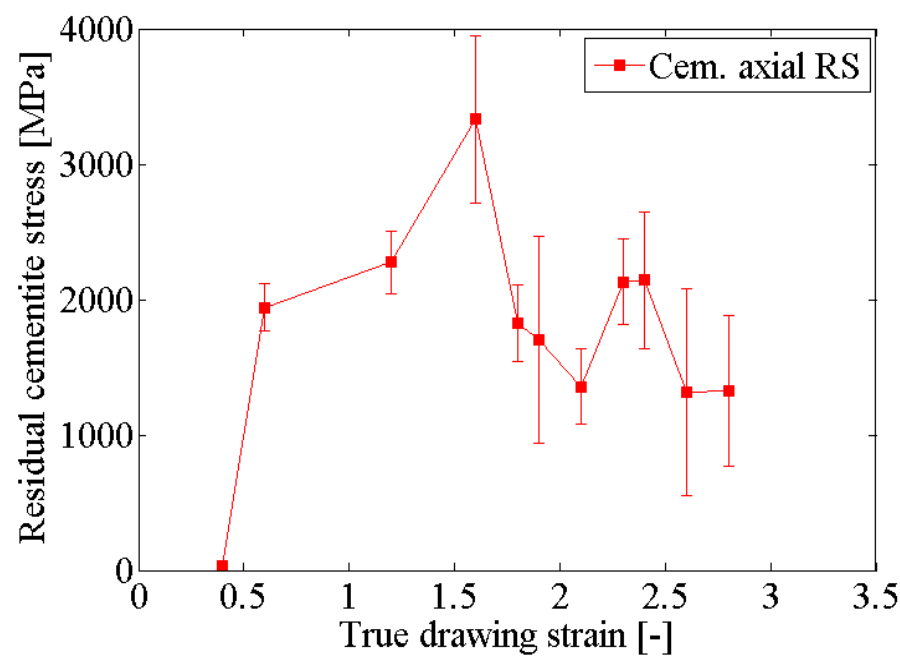

Fig. 5. Axial residual stress in the cementite phase as a function of drawing strain.

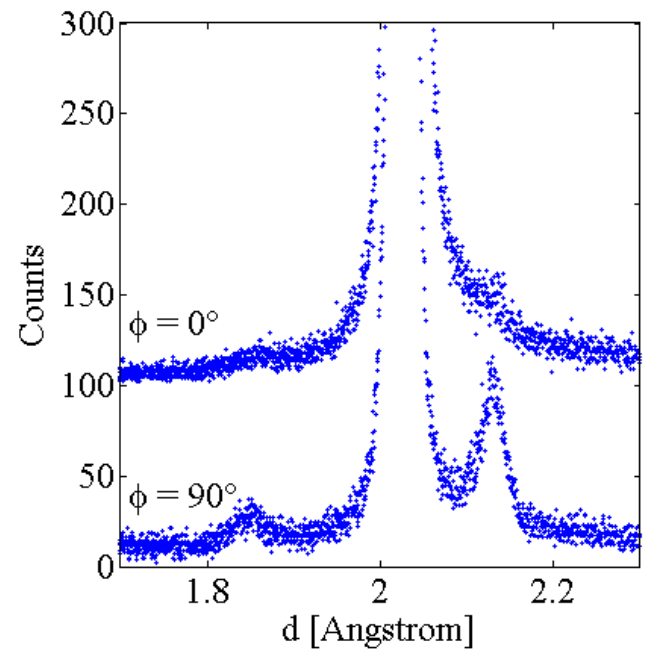

Fig. 6. Detail of diffraction spectra measured on wire with $\varepsilon=2.1\left(\psi=30^{\circ}\right)$.

Intensity distribution The intensity evolution of the cementite peak at $1.85 \AA$ and the brass peak was investigated. To eliminate intensity evolutions related to peak broadening and determine the texture correctly, the integrated intensity should be used. Here the maximum intensity is used because the peak that overlaps with the 110 ferrite reflection could not be fitted and the fit of the other peak was often unreliable. However, because the observed peak width doesn't change (see below), using the maximum intensity should be a good approximation. The point where strong asymmetry begins is chosen as the maximum intensity for the overlapping peak. The observed maximum intensity is corrected by dividing by beam current.

The intensity distribution for two different drawing strains is shown in Fig. 7. for $\varphi=0^{\circ}$ and $90^{\circ}$ on the left and right hand side, respectively. The cementite peak only shows a decreasing intensity towards higher $\psi$ tilts, which is a geometrical effect. At higher strains the intensity also drops visibly. It can thus be concluded that the cementite shows no significant indication of texture.
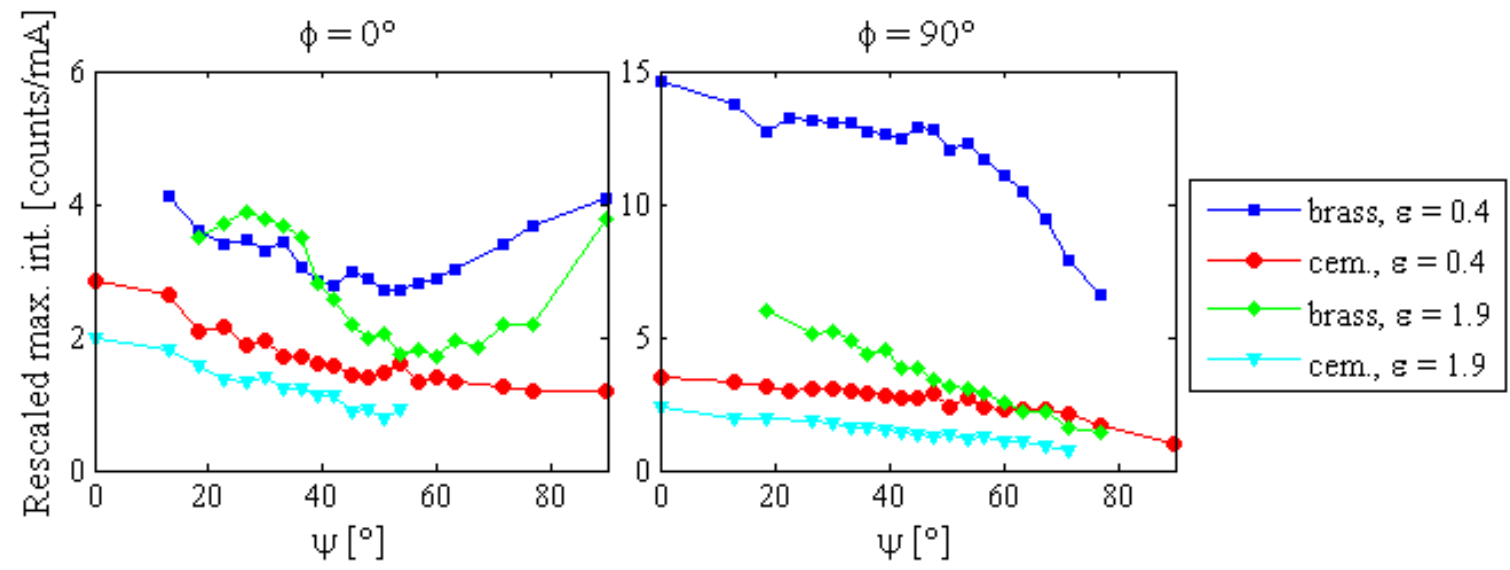

Fig. 7. Rescaled maximum intensity for both $\phi=0^{\circ}$ and $\phi=90^{\circ}$, as a function of $\psi$. This corresponds to the intensity evolution along two lines of a pole figure with the wire axis parallel to the horizontal axis. For $\phi=0^{\circ}$, the horizontal line of the polefigure from the centre to the edge. For $\phi=90^{\circ}$, the veritcal line of the polefigure.

The 111 brass peak on the other hand does show a texture evolution. Along the $\varphi=90^{\circ}$ direction there is no intensity evolution except for a geometrical effect. However, along the $\varphi=0^{\circ}$ direction at a drawing strain of 1.9 there is a clear intensity evolution. At higher strains the same evolution becomes even more pronounced. The observed intensity agrees well with a double fibre texture $<111>+<001\rangle$ as can be expected for a fcc material subjected to axially symmetric elongation. 

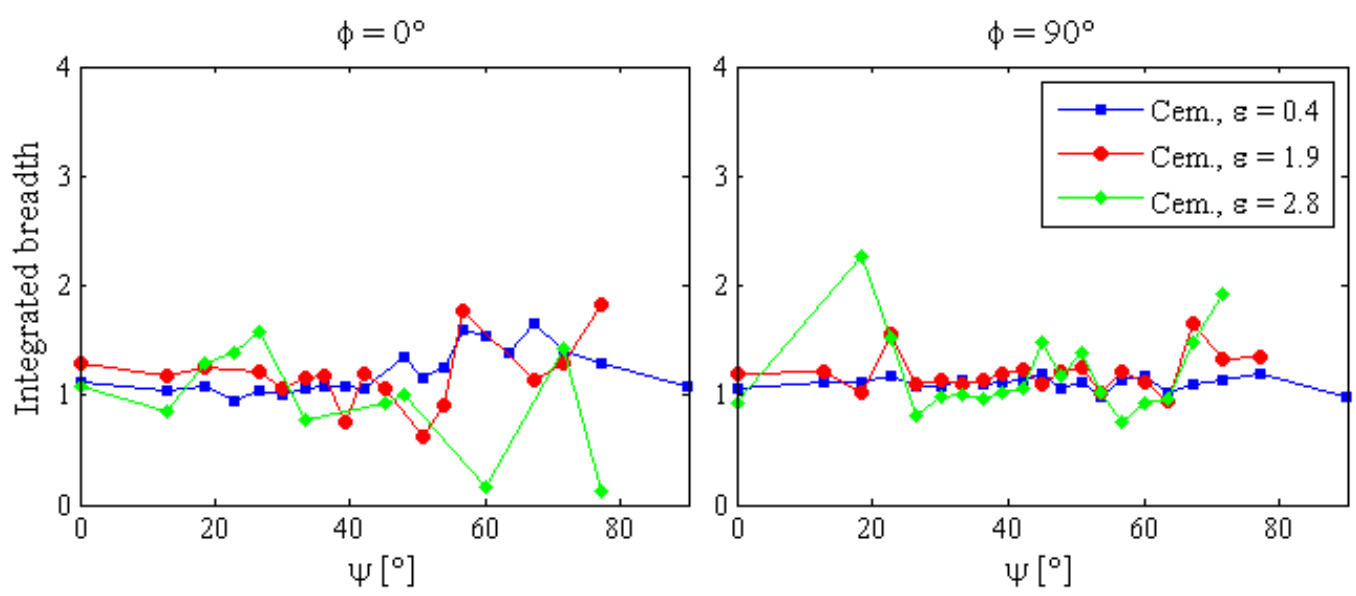

Fig. 8. Integrated peak width as a function of $\psi$ and drawing strain for both $\phi=0^{\circ}$ and $\phi=90^{\circ}$.

Peak broadening The peak width of the only cementite peak that persists to higher strains has been determined. After fitting of the peak the integrated breadth was calculated. The result for three different strains is shown in Fig. 8. for $\varphi=0^{\circ}$ and $90^{\circ}$ on the left and right hand side respectively. No changes in peak width could be observed for strains above 0.4 .

\section{Discussion}

Residual stress The fact that the stress is calculated from a peak consisting of serveral reflections requires some consideration. The $\mathrm{d}_{0}$ spacing is not required because $\mathrm{d}-\sin ^{2} \psi$ curves are used to calculate the residual stresses. However, the diffraction elastic constants (DEC's) to be used in the calculation should be known. This is not straightforward because the DEC's of the three contributing reflections are different. The DEC of the 221 reflection is used because, based on the pattern of the as-patented wire, it gives the largest contribution to the observed peak. The DEC's were calculated according to Kröner and a homogeneous texture was assumed.

One problem that might occur is a fake peak shift being recorded as a consequence of one of the three reflection preferentially increasing in intensity due to texture. Since no significant cementite texture was observed, this problem does not arise here. As long as the stress on the different lattice planes doesn't vary to much as a result of grain-grain interactions, the calculated value for the stress is considered to be a reasonable approximation for the stress in the cementite phase.

It should be remarked that, as a result of the decreasing wire diameter, the sample is only fully penetrated by X-rays starting from $\varepsilon=0.8$. The measured stress therefore evolves from a mixture of macro stress and micro phase stress at $\varepsilon=0$ to only micro phase stress at $\varepsilon=0.8$ because the macro stresses cancel out when averaged over the entire cross-section.

The residual stress in the axial direction increases up to $3.3 \mathrm{GPa}$ at a true drawing strain of 1.6 and then drops and saturates at $1.8 \mathrm{GPa}$. The saturation of the cementite residual stress corresponds to a change in regime observed in the ferrite stress reported elsewhere [14]. The strain at which this happens also seems to coincide with the completion of the ferrite texture and lamellae rotation. Oliver et al. [19] and Hanabusa et al. [20] reported saturation of residual stress for spheroidised cementite. Oliver attributed the saturation to yielding upon unloading. Another possible mechanism for saturation of the cementite stress is fragmentation of the cementite lamellae. The current investigation did not allow to draw conclusions regarding the responsible mechanisms.

$\boldsymbol{a}$-brass The peak having a d-spacing of around $2.13 \AA$ is considered to be the 111 reflection of the brass coating. Its average intensity decreases (see Fig. 7 for $\varphi=90^{\circ}$ ) as a result of the peak 
broadening caused by deformation of the microstructure. It also shows an increasingly strong $<111\rangle+<001\rangle$ fibre texture. The fcc $\alpha$-brass is softer and more ductile then the bulk wire. This can explain the change of peak position between $\varphi=0^{\circ}$ and $90^{\circ}$ as a compressive residual stress in the axial direction. The peak has a higher intensity in all patterns where $\varphi=90^{\circ}$, as a result of the alignment of the wire and the incoming synchrotron beam.

All the above observation form a consistent picture. It is therefore safe to assume that the peak at $2.13 \AA$ is indeed $\alpha$-brass. Moreover the 111 reflection is the strongest reflection for fcc materials, which explains why only the 111 reflection is observed. The fact that the peak persists to high strains makes it very unlikely that this is a cementite peak. Strong deformation of the cementite and its decomposition at high strains should strongly decrease the intensity of the observed reflections.

Cementite texture No indication of a significant cementite texture could be observed. This is in disagreement with neutron diffraction measurements by K. Van Acker et al. [21]. The reason for this could be that the current experiment was not designed to measure texture. However, it is also possible that there is indeed no texture present in the cementite. The cementite is a hard phase which does not easily deform plastically. This will hinder the development of texture.

The development of texture could further be suppressed because of the fine lamellar microstructure. The development of the ferrite fibre texture is also retarded because of this [22]. If a material consisting of a soft and hard phase is deformed, the soft phase has to flow around and adapt to the hard phase. This leads to lattice bending in the soft phase and smearing out of the preferred orientations resulting in a smoothening of the texture. Because of the nature of this mechanisms it might not manifest itself so strongly in the hard phase. The fine lamellar microstructure will result in a large material volume affected by the phase boundaries. Requirements of compatibility between the two phases at the interface and low interface energy might cause bending of the crystal lattice and spreading out of any texture present.

Any potential texture development could also be destroyed by a breakup of the lamellae [7,23] in small disoriented crystallites. Rotations of the crystallites without preferred direction, would destroy the present texture. It should be noted that this was only observed at high strains [8]. A strong dissolution of the cementite could also lead to the suppression or destruction of texture.

Cementite peak broadening Broadening of the cementite peaks could only be observed at the onset of deformation and not at higher strains. This is in disagreement with the observations made in [9]. However, the lack of broadening in the current investigation is probably due to the instrumental broadening being too large to accurately measure the cementite peak profiles. The reduction in peak intensity without a broadening of the peaks indicates that when the peaks broaden, the tails are lost in the background.

A big change in peak widths and intensities can be observed when comparing the profile of the aspatented wire and the one at true drawing strain of 0.4. This is an indication that the cementite is already undergoing plastic deformation at the early stages of wire drawing.

\section{Conclusions}

The observed cementite peaks quickly vanish with increasing strain. Together with the overlapping with the 110 ferrite and 111 brass reflection this results in only one cementite peak being of any value. From this one peak some conclusions could be drawn.

1. The stress in the cementite reaches a maximum of $3.3 \mathrm{GPa}$ at a true strain of 1.6 and drops to a plateau of $1.8 \mathrm{GPa}$ at higher strains. 
2. No significant cementite texture could be observed.

3. No peak broadening could be observed. This can be attributed to the large instrumental peak broadening. The difference in the diffraction profiles between 0 and 0.4 true strain indicates that the cementite might already undergo plastic deformation at small drawing strains.

Although the experiment was aimed at measuring ferrite stresses, a thorough study of the obtained profiles also allowed to learn something about the cementite phase and gather the maximum amount of information.

\section{Acknowledgements}

This work was funded by JT's fellowship of the Fund for Scientific Research-Flanders (FWO-V). The author gratefully acknowledges BESSY at Helmholtz Zentrum Berlin (HZB) for providing beam time and support at EDDI. The authors are grateful for the support from and helpful discussions with M. Klaus and Ch. Genzel from HZB. The authors also gratefully acknowledges support from the Belgian Science Policy BELSPO through the IAP project 7/21 "Multiscale Mechanics of Interface Dominated Materials".

\section{References}

[1] W.F. Hosford Jr., Trans. Metall. Soc. AIME 230 (1964), 12-15

[2] G. Langford, Metallurgical Trans. A 8 (1977), 861-875

[3] J. Gil Sevillano, Journal de Physique III 1 (1991), 967-988

[4] J. Gil Sevillano, A twist on heavily drawn wires, Mordica Lecture, Wire Expo 2010

[5] V.N. Gridnev, V.G. Gavrilyuk, Physics of Metals 4 (1982), 531-551

[6] X. Sauvage, J. Copreaux, F. Danoix, D. Blavette, Philosophical Magazine A 80 (2000), 781-796

[7] J. Languillaume, G. Kapelski, B. Baudelet, Acta Mater. 45 (1997), 1201-1212

[8] H.G. Read, W.T. Reynolds Jr., K. Hono, T. Tarui, Scripta Mater. 37 (1997), 1221-1230

[9] Y.J. Li, P. Choi, C. Borchers, S. Westkamp, S. Goto, D. Raabe, R. Kirchheim, Acta Mater. 59 (2011), 3965-3977

[10] M. Nikolussi, S.L. Shang, T. Gressmann, A. Leineweber, E.J. Mittemeijer, Y. Wang, Z.-K. Liub, Scripta Mater. 59 (2008), 814-817

[11] J. Alkorta, J. Gil Sevillano, Journal of Materials Research 27 (2011), 45-52

[12] J. Gil Sevillano, Mat. Sc. and Eng. 21 (1975), 221-225

[13] W.C. Chiou Jr., E.A. Carter, Surface Science 530 (2003), 87-100

[14] M. Kriška, J. Tacq, M. Seefeldt, In these proceedings.

[15] Ch. Genzel, I. A. Denks, J. Gibmeier, M. Klaus, G. Wagener, Nucl. Instrum. Methods in Phys. Research A 578 (2007), 23 - 33

[16] M. Seefeldt, M. Kriška, J. Tacq, In these proceedings.

[17] M.A. Weisser, A.D. Evans, S. Van Petegem, S.R. Holdsworth, H. Van Swygenhoven, Acta Mater. 59 (2011), 4448-4457

[18] D.F. Rendle, Journal of Forensic Sciences 26 (1981), 343-351

[19] E.C. Oliver, M.R. Daymond, P.J. Withers, Acta Mater. 52 (2004), 1937-1951

[20] T. Hanabusa, J. Fukura, H. Fujiwara, JSME 12 (1969), 931-939

[21] K. Van Acker, J. Root, P. Van Houtte, E. Aernoudt, Acta Mater. 44 (1996), 4039-4049

[22] A. Walentek, X. Hu, M. Seefeldt, P. Van Houtte, Materials Science Forum 495-497 (2005), 369-374

[23] S. Goto, R. Kirchheim, T. Al-Kassab, C. Borchers, Trans. Nonferrous Met. Soc. China 17 (2007), $1129-1138$ 